\title{
Divergence des Fonctionnelles de M. Kac et Diffusion Quantique
}

\author{
Par \\ Bernard Gaveau* et Edmond MAZET**
}

Table

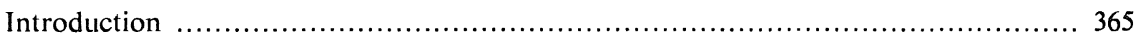

1. Quelques rappels sur la théorie de la diffusion dans un potentiel radial ........... 366

2. Fonction d'onde à énergie nulle et la constante de couplage critique ............. 368

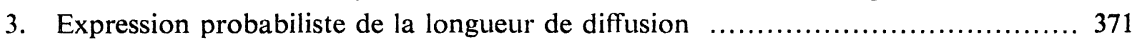

4. Asymptotique des fonctionnelles de Kac si $t \rightarrow \vdash \infty$ et $\lambda, \lambda^{(c)} \ldots \ldots \ldots \ldots \ldots \ldots . . . . . . .372$

5. Ondes partielles de moment cinétique $l$ et leur constante de couplage critique .... 374

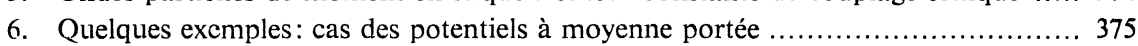

\section{Introduction}

Une particule quantique dans $\mathbb{R}^{3}$ soumise à l'action d'un potentiel $\lambda q$ admet l'hamiltonien $(1 / 2) \Delta+\lambda q$. Ici $\lambda$ désigne une constante de couplage que nous ferons varier par la suite, $\Delta$ est le laplacien de $\mathbb{R}^{3}, q$ est une fonction que nous supposons radiale dans cet article. L'évolution de la particule est donnée par le propagateur résolvant l'équation de Schrödinger et ce propagateur admet une expression formelle comme une intégrale de Feynmann. Cette expression formelle, acquiert un sens comme intégrale de Wiener lorsque l'on considère l'équation de la chaleur associée plutôt que l'équation de Schrödinger. Dans ce cas la résolution de l'équation de la chaleur fait intervenir une fonctionnelle de $\mathrm{Kac}_{x}\left(\exp \left(\lambda \int_{0}^{t} q\left(b_{s}\right) d s\right) f\left(b_{t}\right)\right)$ où $b_{s}$ est le mouvement brownien de $\mathbb{R}^{3}$ issu de $x$ à $s=0$ et $f$ est la donnée initiale (voir [5]), tout au moins tant que les expressions écrites sont des intégrales convergentes. En ce qui concerne certaines quantités d'intérêt physique (valeur propre, asymptotique semi-classique) on peut dire que l'intégrale de Feynmann-Kac-Wiener rend les mêmes services que

Communiqué par H. Araki, le 2 avril, 1981. Revu le 19 mai, 1981.

* Tour Béryl, 40 avenue d'italie, 75013 Paris, France.

** 65 avenue de l'Amiral Mouchez, 75013 Paris, France. 
l'intégrale de Feynmann. Récemment, Kac [6] a montré qu'on pouvait aussi interpréter de façon probabiliste certains aspects de la théorie de la diffusion quantique (par exemple le "scattering inverse", la longueur de diffusion, la mesure spectrale).

Un problème important est donc de donner des conditions de convergence ou de divergence de telles fonctionnelles; des critères de convergence ont été donnés dans [1], [3] ainsi que [7] (où les critères sont combinés avec les inégalités de Harnack) et l'idée de la démonstration a été plus tard reprise par divers auteurs; la méthode consistait à développer l'exponentielle en série et à majorer terme à terme de façon grossière. Nous nous proposons ici de donner une autre méthode d'estimation dans le cas d'un potentiel radial (encore qu'une partie de la méthode puisse s'appliquer au cas inhomogène mutatis mutandis).

Nous montrerons par des exemples que cette méthode nouvelle donne des résultats beaucoup plus précis que ceux de [1] et [3].

Ces résultats ont été partiellement annoncés dans une Note aux Comptes Rendus de l'Académie des sciences de Paris [4].

Nous remercions le referee de cet article pour les remarques et les précisions qu'il nous a données.

\section{Quelques Rappels sur la Théorie de la Diffusion dans un Potentiel Radial}

a) Nous considérons ici l'équation de Schrödinger dans $\mathbb{R}^{3}$, dans un potentiel radial $\lambda q(r)$ ( $r$ étant la distance à l'origine); $\lambda$ est une constante de couplage que nous ferons varier. Cette équation, à l'énergie $-k^{2}$ est donc

$$
\frac{1}{2} \Delta \psi+\lambda q \psi=-k^{2} \psi
$$

Si nous supposons $\psi$ radiale, nous pouvons nous ramener à une équation de Sturm-Liouville classique en posant $\tilde{\psi}(r)=r \psi(r)$, de sorte que (1.1) devient dans ce cas

$$
\left\{\begin{array}{l}
\frac{1}{2} \tilde{\psi}^{\prime \prime}+\lambda q \tilde{\psi}=-k^{2} \tilde{\psi} \\
\tilde{\psi}(0)=0
\end{array}\right.
$$

Nous allons supposer ici que $q(r)$ est un potentiel à courte portée, ce qui signifiera pour nous que 


$$
\int_{0}^{+\infty}|q(r)| r d r<+\infty .
$$

b) Dans ces conditions, on peut appliquer la théorie de la diffusion classique; en particulier (1.2) admet deux solutions $y_{\lambda}^{(1)}\left(x,-k^{2}\right)$ et $y_{\lambda}^{(2)}\left(x,-k^{2}\right)$ avec

$$
\begin{cases}y_{\lambda}^{(1)}\left(0,-k^{2}\right)=0 & y_{\lambda}^{(2)^{\prime}}\left(0,-k^{2}\right)=1 \\ y_{\lambda}^{(1)^{\prime}}\left(0,-k^{2}\right)=1 & y_{\lambda}^{(2)^{\prime}}\left(0,-k^{2}\right)=0 .\end{cases}
$$

Dans ces conditions, le comportement asymptotique de $y_{\lambda}^{(1)}\left(x,-k^{2}\right)$ est

$$
y_{\lambda}^{(1)}\left(x,-k^{2}\right) \sim \frac{1}{-2 i k}\left[f_{\lambda}(-k) e^{i \sqrt{2} k x}-f_{\lambda}(k) e^{-i \sqrt{2} k x}\right]
$$

et de même pour $y_{\lambda}^{(2)}\left(x,-k^{2}\right)$ en remplaçant $f_{\lambda}(k)$ par $g_{\lambda}$.

\section{Nous supposerons}

$$
f_{\lambda} \text { et } g_{\lambda} \text { sont holomorphes de } k \text { près de } k=0
$$

L'hypothèse (1.3) n'est pas suffisante en général pour assurer (1.5); mais pour $q$ à support compact, ou superposition de potentiels de Yukawa, (1.5) est réalisée.

Comme le wronskien de $\left(y_{\lambda}^{(1)}, y_{\lambda}^{(2)}\right)$ est constant et vaut 1 en $x=0$, si nous comparons sa valeur en $x=\infty$ nous déduisons de (1.5) et de son analogue $y_{\lambda}^{(2)}$

$$
1=\frac{1}{2 k}\left(f_{\lambda}(i k) g_{\lambda}(-i k)-f_{\lambda}(-i k) g_{\lambda}(i k)\right)
$$

En particulier, de la formule (1.6) et de l'holomorphie des fonctions pour les valeurs $k=0$, nous déduisons

Lemme 1. Seulement l'une des deux quantités $f_{\lambda}(i k)$ ou $g_{\lambda}(-i k)$ peut s'annuler à l'ordre 1 en $k=0$ (ce qui ne veut pas dire qu'elle s'annule effectivement).

c) Les états liés sont les valeurs $k$ imaginaires pures tels que (1.1) ait une solution $\psi$ de carré intégrable. Cela signifie donc que pour ces valeurs de $k$, $y_{\lambda}^{(1)}\left(0,-k^{2}\right)$ est de carré intégrable et donc que $f_{\lambda}(k)$ acquiert un zéro dans le demi-plan supérieur (pour le moment, nous ne regardons que les états liés de moment cinétique 0 ).

d) Le cas des solutions à énergie nulle $(k=0)$, doit être considéré à part. On peut montrer facilement que si $f_{\lambda}(0) \neq 0$, il existe une unique solution $\tilde{v}_{\lambda}$ de (1.7) 


$$
\left\{\begin{array}{l}
\frac{1}{2} \tilde{v}_{\lambda}^{\prime \prime}+\lambda q \tilde{v}_{\lambda}=0 \\
\tilde{v}_{\lambda}(0)=0 \\
\tilde{v}_{\lambda}(x) \sim x-l_{\lambda} \quad \text { si } \quad x \rightarrow+\infty .
\end{array}\right.
$$

En particulier $\tilde{v}_{\lambda}(x)=y_{\lambda}^{(1)}(x, 0)\left(f_{\lambda}(0)\right)^{-1} ; l_{\lambda}$ est appelée la longueur de diffusion du potentiel $\lambda q$. C'est un invariant de la diffusion à basse énergie.

Lorsque $f_{\lambda}(0)=0$, on peut trouver une solution de (1.7) mais avec $\tilde{v}_{\lambda}(x)$ $\sim 1$ si $x \rightarrow+\infty$.

e) Définissons maintenant la fonction spectrale en admettant tout d'abord qu'il n'y ait pas d'états liés. Alors on a d'après (1.5)

$$
y_{\lambda}^{(1)}\left(x,-k^{2}\right) \sim \alpha\left(k^{2}\right) \sin \left(\sqrt{2} k x+\delta\left(k^{2}\right)\right)
$$

où $\delta\left(k^{2}\right)$ est le déphasage et $\alpha\left(k^{2}\right)$ l'amplitude. Lorsqu'il y a des états liés, $k^{2}$ devient négatif et $y_{\lambda}^{(1)}\left(x,-k^{2}\right)$ est la fonction propre nulle en 0 de carré intégrable. La fonction spectrale $\rho_{\lambda}\left(k^{2}\right)$ est celle que

$$
\int_{-\infty}^{+\infty} y_{\lambda}^{(1)}\left(x,-k^{2}\right) y_{\lambda}^{(1)}\left(x^{\prime},-k^{2}\right) d \rho_{\lambda}\left(k^{2}\right)=\delta\left(x^{\prime}-x\right)
$$

et $\rho_{\lambda}\left(k^{2}\right)$ est fonction croissante ayant des sauts discrets en les états liés. Si $L$ désigne l'opérateur $(1 / 2)\left(d^{2} / d x^{2}\right)+\lambda q$, on a la décomposition de Stone-VonNeumann dans ce cas

$$
\left(L^{\alpha} f, g\right)=\int\left(k^{2}\right)^{\alpha}\left(\int f(x) y_{\lambda}^{(1)}\left(x,-k^{2}\right) d x\right)\left(\int g(x) y_{\lambda}^{(1)}\left(x,-k^{2}\right) d x\right) d \rho_{\lambda}\left(k^{2}\right) .
$$

(1.9) bis $\left(e^{+L t} f, g\right)$

$$
=\int e^{-k^{2} t}\left(\int f(x) y_{\lambda}^{(1)}\left(x,-k^{2}\right) d x\right)\left(\int g(x) y_{\lambda}^{(1)}\left(x,-k^{2}\right) d x\right) d \rho_{\lambda}\left(k^{2}\right) .
$$

On démontre, par ailleurs, que pour $k^{2} \geqq 0$, on a

$$
d \rho_{\lambda}\left(k^{2}\right)=\frac{1}{\pi}\left(\frac{d\left(k^{2}\right)}{\alpha_{\lambda}^{2}\left(k^{2}\right) k}\right) \quad(\text { voir [6]) }
$$

Dans le cas d'une particule libre $(q=0)$ on a

$$
\rho_{0}\left(k^{2}\right)=\frac{1}{3 \pi}\left(k^{2}\right)^{3 / 2} \text {. }
$$

\section{Fonction d'Onde à Énergie Nulle el la Constante de Couplage Critique}

Notons maintenant $v_{\lambda}$ la solution de (1.1) radiale, avec $k^{2}=0$ telle que 
$v_{\lambda}(r) \rightarrow 1$ si $r \rightarrow+\infty$. Alors $\tilde{v}_{\lambda}(r)=r v_{\lambda}(r)$ est la fonction satisfaisante (1.7).

Notons par ailleurs $b_{t}$ le mouvement brownien standard de $\boldsymbol{R}^{3} ; \mathrm{E}_{\boldsymbol{x}}$ désignera l'espérance conditionnelle sachant que $b_{0}=x\left(x \in \boldsymbol{R}^{3}\right)$. L'équation de la chaleur

$$
\left\{\begin{array}{l}
\frac{\partial f}{\partial t}=\frac{1}{2} \Delta f+\lambda q f \\
f(0, x)=\varphi(x)
\end{array}\right.
$$

$x$ résout par la formule de $\mathrm{M}$. Kac [5]

$$
f(t, x)=\mathrm{E}_{x}\left(\exp \left(\lambda \int_{0}^{t} q\left(b_{s}\right) d s\right) \varphi\left(b_{t}\right)\right)
$$

lorsque toutes les intégrales écrites sont convergentes. Par ailleurs, il a été démontré dans [1], le théorème suivant:

Théorème 1.1. Si q satisfait la condition de courte portée (1.3) on a

$$
\mathrm{E}_{x}\left(\exp \left(\lambda \int_{0}^{+\infty} q\left(\left|b_{s}\right|\right) d s\right)\right)<+\infty
$$

pour $\lambda$ assez petit.

Nous supposerons désormais que $q \geqq 0$ car les parties négatives de $q$ ne font qu'améliorer les convergences des exponentielles de Kac. Nous avons alors le lemme évident suivant:

Lemme 2.2. La quantité $M_{t} \equiv \exp \left(\lambda \int_{0}^{t} q\left(\left|b_{s}\right|\right) d s\right) v_{\lambda}\left(b_{t}\right)$ est une martingale locale (c'est à dire que si $T_{R}$ désigne le temps de sortie de la boule de centre 0 et de rayon $R$, alors $M_{t \Lambda T_{R}}$ est une martingale).

La démonstration est de routine en tenant compte du fait que $v_{\lambda}$ est solution $(1 / 2) \Delta v_{\lambda}+\lambda q v_{\lambda}=0$.

Théorème 2.3. Pour que $\mathrm{E}_{x}\left(\exp \left(\lambda \int_{0}^{+\infty} q\left(\left|b_{s}\right|\right) d s\right)\right)$ soit fini, il faut et il suffit que la fonction d'onde d'énergie nulle qui tend vers 1 à l'infini, $v_{\lambda}(x)$ ne s'annule pas et on a alors

$$
v_{\lambda}(x)=\mathrm{E}_{x}\left(\exp \left(\lambda \int_{0}^{+\infty} q\left(\left|b_{s}\right|\right) d s\right)\right) .
$$

De plus, le premier $\lambda$ tel que $\mathrm{E}_{0}\left(\exp \left(\lambda \int_{0}^{+\infty} q\left(\left|b_{s}\right|\right) d s\right)\right)$ diverge est le premier pôle de la fonction méromorphe $\lambda \rightarrow f_{\lambda}(0)^{-1}$ et nous l'appelerons première constante de couplage critique et la noterons $\lambda_{1}^{(c)}$.

Démonstration. La quantité $M_{t \Lambda T_{R}}$ est une martingale. 
a) Si $v_{\lambda}$ ne s'annule pas, elle est positive et donc a une limite si $t \rightarrow+\infty$ et la limite est de nouveau une surmartingale positive $M_{T_{R}}$ (par rapport à la famille de temps d'arrêt $T_{R}$ ), par conséquent elle a une limite (théorème de Doob [2]). Comme $v_{\lambda}\left(b_{t}\right) \rightarrow 1$ si $t \rightarrow+\infty$ par définition de $v_{\lambda}$ on a d'après le lemme de Fatou que

$$
\mathrm{E}_{x}\left(\exp \left(\lambda \int_{0}^{+\infty} q\left(\left|b_{s}\right|\right) d s\right)\right) \leqq v_{\lambda}(x)
$$

Mais alors $\exp \left(\lambda \int_{0}^{t} q\left(\left|b_{s}\right|\right) d s\right) v_{\lambda}\left(b_{t}\right)=M_{t}$ reste dominée par une fonctionnelle intégrable donc $M_{t}$ est une vraie martingale dominée par une fonctionnelle intégrable; le théorème de Doob nous apprend alors que $M_{t}$ a une limite qui ne peut être que $\exp \left(\lambda \int_{0}^{+\infty} q\left(\left|b_{s}\right|\right) d s\right)$ qui est intégrable et que de plus

$$
v_{\lambda}(x)=\mathrm{E}_{x}\left(\exp \left(\lambda \int_{0}^{t} q\left(\left|b_{s}\right|\right) d s\right) v_{\lambda}\left(b_{t}\right)\right)=\mathrm{E}_{x}\left(\exp \left(\lambda \int_{0}^{+\infty} q\left(\left|b_{s}\right|\right) d s\right)\right)
$$

b) réciproquement si $\mathrm{E}_{x}\left(\exp \left(\lambda \int_{0}^{+\infty} q\left(\left|b_{s}\right|\right) d s\right)\right)$ est fini pout tout $x$, on voit aussitôt que $M_{t}$ est une vraie martingale et donc (2.5) a lieu; en particulier $v_{\lambda}$ est positive.

c) D'après le théorème 2.1 , pour $\lambda>0$ assez petit, $\mathrm{E}_{x}\left(\exp \left(\lambda \int_{0}^{+\infty} q\left(\left|b_{s}\right|\right) d s\right)\right)$ est fini. Par ailleurs, comme nous avons supposé $q \geqq 0$, cette quantité est finie sur un intervalle $\left[0, \lambda_{1}^{(c)}\right]$ et divergera après; ce $\lambda_{1}^{(c)}$ est tel que $\lambda>\lambda_{1}^{(c)}, v_{\lambda}$ ne soit plus positive; $\lambda_{1}^{(c)}$ est donc le premier $\lambda$ pour lequel $\tilde{v}_{\lambda}$ est asymptotique à 1 ; donc c'est effectivement le premier zéro de $f_{\lambda}(0)$ (voir $\left.1, \mathrm{~d}\right)$ ). C'est encore le premier $\lambda$ pour lequel $\lambda q$ a un état lié qui est 0 .

Evidemment pour $\lambda>\lambda_{1}^{(c)}$, l'exponentielle de Kac diverge. Soit d'autre part $T_{R}$ le premier temps de sortie de la boule de centre 0 et de rayon $R$ dans $\boldsymbol{R}^{3}$.

Théorème 2.4. Soit $\lambda>\lambda_{1}^{(c)}$ et $R_{\lambda}$ la position du premier zéro de $v_{\lambda}$. Alors pour $x$ tel que $|x|<R<R_{\lambda}$, on a

$$
\mathbb{E}_{x}\left(\exp \left(\lambda \int_{0}^{T_{R}} q\left(\left|b_{s}\right|\right) d s\right)\right)<+\infty
$$

et

$$
\mathbb{E}_{x}\left(\exp \left(\lambda \int_{0}^{T_{R_{\lambda}}} q\left(\left|b_{s}\right|\right) d s\right)\right)=+\infty
$$

De plus, on a 


$$
\lim _{t \rightarrow+\infty} \frac{\log \mathrm{E}_{x}\left(\exp \lambda \int_{0}^{T_{R_{\lambda}} A t} q\left(\left|b_{s}\right|\right) d s\right)}{t}=0
$$

Démonstration. Reprenons la martingale $M_{t}$ du lemma 2.2. Il est clair que $M_{t \Lambda T_{R}}$ est une martingale de signe constant et toujours par le théorème de Doob elle a une limite et on a

$$
\mathrm{E}_{x}\left(\exp \left(\lambda \int_{0}^{T_{R}} q\left(\left|b_{s}\right|\right) d s\right)\right) \leqq v_{\lambda}(R)^{-1}
$$

Si $\exp \left(\lambda \int_{0}^{T_{R_{\lambda}}} q\left(\left|b_{s}\right|\right) d s\right)$ était intégrable, $M_{t \Lambda T_{R_{\lambda}}}$ serait alors dominée par une fonctionnelle intégrable, on tirerait alors d'après le théorème de Doob,

$$
v_{\lambda}(x)=\mathrm{E}_{x}\left(\lim _{t \rightarrow+\infty} M_{t \Lambda T_{R_{\lambda}}}\right)=\mathrm{E}_{x}\left(M_{T_{R_{\lambda}}}\right) .
$$

On a $v_{\lambda}(x) \neq 0$ et $v\left(b_{T_{R_{\lambda}}}\right)=0$, d'où contradiction.

Enfin considérons d'opérateur $(1 / 2)\left(d^{2} / d x^{2}\right)+\lambda q$ sur $\left[0, R_{\lambda}\right]$ avec condition au bord nulles en 0 et $R_{\lambda}$. Alors, $\tilde{v}_{\lambda}$ est précisement une fonction propre de valeur propre 0; par suite l'estimée de Kac [5] sur le calcul de la première valeur propre nous donne le résultat attendu.

\section{Une Expression Probabiliste de la Longueur de Diffusion}

Donnons maintenant très rapidement, une expression probabiliste de $l_{\lambda}$ un peu différente de celle trouvée par Kac [6].

Théorème 3.1. La longueur de diffusion $l_{\lambda} d u$ potentiel $\lambda q$ pour $\lambda<\lambda_{1}^{(c)}$ est donnée par

$$
4 \pi l_{\lambda}=\lim _{R \rightarrow+\infty} \frac{1}{R^{2}} \int_{|x| \leqq R}\left(1-\mathrm{E}_{x}\left(\exp \left(\lambda \int_{0}^{+\infty} q\left(\left|b_{s}\right|\right) d s\right)\right)\right) d x .
$$

Démonstration. On utilise le fait que

$$
v_{\lambda}(r) \sim\left(1-\frac{l_{\lambda}}{r}\right) \quad(\text { voir }(1.7))
$$

et le calcul (2.4) de $v_{\lambda}(r)$, d'où

$$
1-\mathrm{E}_{x}\left(\exp \left(\lambda \int_{0}^{+\infty} q\left(\left|b_{s}\right|\right) d s\right)\right) \sim \frac{l_{\lambda}}{r} .
$$




\section{Asymptotique des Fonctionnelles de Kac pour $t \rightarrow+\infty$ et $\lambda>\lambda_{1}^{(0)}$}

Théorème 4.1. Pour $\lambda=\lambda_{1}^{(c)}$ et $t \rightarrow+\infty$, on $a$

$$
\mathrm{E}_{x}\left(\exp \left(\lambda_{1}^{(c)} \int_{0}^{t} q\left(\left|b_{s}\right|\right) d s\right)\right) \sim C t^{1 / 2},
$$

et pour $\lambda>\lambda_{1}^{(c)}, t \rightarrow+\infty$, on $a$

$$
\mathrm{E}_{x}\left(\exp \left(\lambda \int_{0}^{t} q\left(\left|b_{s}\right|\right) d s\right)\right) \sim C e^{\mu(\lambda) t}
$$

où $\mu(\lambda)$ est la valeur de l'énergie du premier état lié du potentiel $\lambda q$ et $C$ sont des constantes indépendantes de $t$.

Preuve. Comme dans [6], introduisons la transformée de Laplace

$$
\hat{u}_{s}(x)=s \int_{0}^{+\infty} e^{-s t} \mathrm{E}_{x}\left(\exp \left(\lambda \int_{0}^{t} q\left(\left|b_{s}\right|\right) d s\right)\right) d t
$$

Lorsque $\hat{u}_{s}$ converge, elle satisfait

$$
\left\{\begin{array}{l}
\frac{1}{2} \Delta \hat{u}_{s}+(\lambda q-s) \hat{u}_{s}=-s \\
\hat{u}_{s}(\infty)=1 .
\end{array}\right.
$$

Si nous notons $\varphi_{s}$ une solution particulière de (4.4), nous avons

$$
\tilde{u}_{s}(x)=\varphi_{s}(x)+C_{1} y_{\lambda}^{(1)}(x, s)+C_{2} y_{\lambda}^{(2)}(x, s) .
$$

Or

$$
\tilde{u}_{s}(x) \sim x \quad \text { si } x \rightarrow+\infty,
$$

donc

$$
C_{1} f_{\lambda}(-i \sqrt{s})+C_{2} g_{\lambda}(-i \sqrt{s})=0
$$

d'après (4.5) et (1.5); de plus

$$
\begin{array}{ll} 
& \varphi_{s}(0)+C_{2}=0 \\
\text { d'où } & \tilde{u}_{s}^{\prime}(0)=\varphi_{s}^{\prime}(0)+\frac{g_{\lambda}(-i \sqrt{s})}{f_{\lambda}(-i \sqrt{s})} \varphi_{s}(0) .
\end{array}
$$

Or d'après le théorème 2.2 , si $\lambda=\lambda_{1}^{(c)}, 0$ est zéro de $f_{\lambda_{1}^{(c)}}(-i \sqrt{s})$. Par le lemme 1.1 , il n'est pas zéro de $g_{\lambda(c)}(-i \sqrt{s})$ et il est zéro simple de $f_{\lambda(c)}$, d'où $\tilde{u}_{s}^{\prime}(0)$ est en $s^{-1 / 2}$ si $s \rightarrow 0$, d'où $\hat{u}_{s}(0)$ est aussi en $s^{-1 / 2}$ et par conséquent, d'après le théorème taubérien de Wiener appliqué à la transformée de Laplace (4.3), nous déduisons (4.1). 
Si $\lambda>\lambda_{1}^{(c)}, v_{\lambda}$ a alors un zéro et $\lambda q$ a alors un état lié $\mu(\lambda)>0$; on conclut alors l'estimée (4.2) par la formule de Kac [5].

Nous allons maintenant préciser davantage le comportement asymptotique des fonctionnelles de $\operatorname{Kac}(4.2)$ pour $\lambda>\lambda_{1}^{(c)}$; dans ce cas si $\lambda$ est assez voisin de $\lambda_{1}^{(c)}$, il n'existe qu'un seul état lié pour $\lambda q$, d'énergie $\mu(\lambda)$ et de fonction propre de carré intégrable $\psi_{\lambda}$. Posons alors

$$
\varphi_{t}(r)=\int\left(p_{t}^{(\mathrm{rad})}\left(r, r^{\prime}\right)-e^{\mu(\lambda) t} \psi_{\lambda}(r) \psi_{\lambda}\left(r^{\prime}\right)\right) d r^{\prime}
$$

où $P_{t}^{(\mathrm{rad})}\left(r, r^{\prime}\right)$ désigne le noyau de la chaleur de l'équation (2.1) mais restreint seulement aux fonctions radiales dans $\boldsymbol{R}^{3}$; sous le signe d'intégrale dans (4.6) ne figure donc que la contribution du spectre continu négatif de (2.1) au calcul du noyau de la chaleur radiale puisqu'on a retranché la partie discrète du spectre $e^{\mu(\lambda) t} \psi_{\lambda}(r) \psi_{\lambda}\left(r^{\prime}\right)$

Posons toujours $\hat{\varphi}_{s}(r)=s \int_{0}^{+\infty} e^{-s t} \varphi_{t}(r) d t$ et $\tilde{\varphi}_{s}(r)=r \hat{\varphi}_{s}(r)$ de sorte que

$$
\begin{aligned}
& \tilde{\varphi}_{s}^{\prime \prime}(r)+(\lambda q-s) \tilde{\varphi}_{s}=-s\left(1-\psi_{\lambda}(r)\left(1 \mid \psi_{\lambda}(r)\right)\right) r \\
& \tilde{\varphi}_{s}(0)=0 \\
& \tilde{\varphi}_{s}(r) \sim r, \quad r \longrightarrow+\infty .
\end{aligned}
$$

Si nous reprenons alors la démonstration du théorème 4.1, nous voyons que

$$
\mathrm{E}_{x}\left(\exp \left(\lambda \int_{0}^{t} q\left(\left|b_{s}\right|\right) d s\right)\right)-e^{\mu(\lambda) t} \psi_{\lambda}(x)\left(1 \mid \psi_{\lambda}\right)
$$

reste borné tant que $\lambda<\lambda_{1}^{(c)}$ où $\lambda_{1}^{(c)}$ désigne le second zéro de $f_{\lambda}(0)$ et pour $\lambda=\lambda_{1}^{(c)}$, cette quantité divergera comme $\mathrm{Ct}^{1 / 2}$, d'où

Théorème 4.2. Pour $\lambda_{1}^{(c)}<\lambda<\lambda_{2}^{(c)}$, si $t \rightarrow+\infty$, on $a$

$$
\mathrm{E}_{x}\left(\exp \left(\lambda \int_{0}^{t} q\left(\left|b_{s}\right|\right) d s\right)\right)=e^{\mu(\lambda) t} \psi_{\lambda}(x)\left(1 \mid \psi_{\lambda}\right)+0(1)
$$

et pour $\lambda=\lambda_{2}^{(c)}$ on $a$

$$
\mathrm{E}_{x}\left(\exp \left(\lambda_{2}^{(c)} \int_{0}^{t} q\left(\left|b_{s}\right|\right) d s\right)\right)-e^{\mu(\lambda) t} \psi_{\lambda}(x)\left(1 \mid \psi_{\lambda}\right) \sim C \sqrt{t}
$$

où $C$ est indépendante de $t$.

Remarque. Nous voyons que pour $\lambda<\lambda_{1}^{(c)}$, les fonctionnelles de Kac sont bornées, pour $\lambda>\lambda_{1}^{(c)}$, elles divergent exponentiellement et pour $\lambda=\lambda_{1}^{(c)}$, elles divergent en $t^{1 / 2}$. L'exposant de divergence critique $1 / 2$ est donc universel 
dans la catégorie des potentiels à courte portée. Cela présente quelques analogies avec une transition de phase.

Nous verrons plus loin que cela n'est plus le cas pour les potentiels à moyenne portée.

Etudions maintenant la densité spectrale $\rho_{\lambda}(k)$ près de 0 .

Théorème 4.3. Pour $\lambda<\lambda_{1}^{(c)}, \lambda_{1}^{(c)}<\lambda<\lambda_{2}^{(c)}$

$$
\begin{aligned}
& \frac{d \rho_{\lambda}\left(k^{2}\right)}{d\left(k^{2}\right)} \sim C k \quad \text { pour } k \longrightarrow 0^{+} \\
& \text {pour } \lambda=\lambda_{1}^{(c)}, \text { ou } \lambda=\lambda_{2}^{(c)}, \quad \text { on } a: \\
& \frac{d \rho_{\lambda(c)}\left(k^{2}\right)}{d k^{2}} \sim \frac{C}{k} \quad \text { pour } \quad k \longrightarrow 0^{+}
\end{aligned}
$$

ici $C$ est une constante indépendante de $k$.

Démonstration. Les estimées peuvent se montrer par la théorie spectrale usuelle du 1. Ou bien se déduire du comportement asymptotique du noyau de la chaleur lorsque $t \rightarrow+\infty$ grâce aux estimées établies aux théorèmes 4.1 , 4.2, au théorème taubérien de Wiener et à la formule (1.9) (bis).

\section{Ondes Partielles de Moment Cinétique $l$ et leur Constante de Couplage Critique}

Notons $Y_{l, m}$ les harmoniques sphériques usuelles de $\mathbb{R}^{3}$. On peut se borner au cas $Y_{l, m}=P_{l}(\cos \theta)$ où $P_{l}$ est le lième polynôme de Legendre en $\cos \theta$. ( $\theta$ angle du rayon vecteur avec l'axe des $z$ par exemple.)

Soit $v_{l}(t, r) P_{l}(\cos \theta)$ solution de l'équation de la chaleur (2.1) avec donnée initiale $P_{l}(\cos \theta)$ est $u_{l}(t, r)=r v_{l}(t, r)$. Notons

$$
\tilde{u}_{s, l}(r)=s \int_{0}^{+\infty} e^{-s t} u_{l}(r, t) d t
$$

Alors il est classique que $\tilde{u}_{s, l}$ satisfait

$$
\tilde{u}_{s, l}^{\prime \prime}-\left(\frac{l(l+1)}{r^{2}}-\lambda q+s\right) \tilde{u}_{s, l}=-s r .
$$

De plus, on a si $|x|=r$

$$
v_{l}(r, t)=\mathrm{E}_{x}\left(\exp \left(\int_{0}^{t} \lambda q\left(\left|b_{s}\right|\right)-\frac{l(l+1)}{\left|b_{s}\right|^{2}} d s\right) .\right.
$$

La théorie de la diffusion en moment cinétique $l$ est analogue à celle du 1 
à quelques petites modifications près. La différence importante est dans (1.5) et par suite (1.6) où nous avons $k^{2 l+1}$ au dénominateur au lieu de $k$. Un raisonnement parallèle à celui du théorème 4.1 , nous donnera alors

Théorème 5.1. Soit $\lambda_{1, l}^{(c)}$ le premier $\lambda$ tel que l'intégrale donnant $v_{l}(r, t)$ diverge si $t \rightarrow+\infty$. Alors pour cette valeur de $\lambda=\lambda_{1}^{(c)}$, , nous avons

$$
v_{l}(r, t) \sim C t^{l+(1 / 2)}(t \longrightarrow+\infty)
$$

si $\lambda>\lambda_{1, l}^{(c)}$, on a un état lié pour le moment cinétique $l$ et la divergence est exponentielle.

\section{Quelques Examples; Cas des Potentiels à Moyenne Portée}

a) Exemples de calculs explicites pour les potentiels à courte portée.

Nous allons montrer que l'estimation obtenue au théorème 2.2 est plus fine que celle du théorème 2.1, qui, rappelons-le, avait été obtenue par une majoration un peu brutale de l'exponentielle développée en série et estimée terme à terme. Prenons le cas du puits carré: $q(r)=\mathbb{H}_{[0, R]}(r)$.

Le critère de [1] donne $\lambda R^{2} / 2<1$ seulement. Ici le $\lambda$ critique est le premier pour lequel apparaît un état lié, c'est donc le $\lambda$ tel que $\sqrt{\lambda R}=\pi / 2$. De même, prenons $q(r)=\exp (-r)$. Le critère de [1] donne $\lambda<1$. On a ici la fonction de Jost à énergie nulle $J_{0}(2 \sqrt{\lambda})$ et donc nous pouvons autoriser $\lambda<z_{0}^{2} / 4$ où $z_{0}$ est le premier zéro de $J_{0}$ i.e. $z_{0} \sim 2,404$ [8].

b) Exemple de potentiels à moyenne portée. Nous allons montrer sur des exemples explicitement calculables que, lorsque la condition (1.3) de courte portée n'est pas satisfaite, alors la situation de convergence ou divergence des fonctionnelles de $\mathrm{Kac}$ devient assez confuse et, en tout cas, qu'elle mériterait une étude détaillée séparée. Considérons d'abord le potentiel

$$
q(r)=\frac{A}{(1+r)^{2}}
$$

Le critère (1.3) n'est plus vrai (il est juste à la limite). L'exponentielle de Kac n'est plus intégrable car

$$
\mathrm{E}_{0}\left(\exp \int_{0}^{+\infty} \frac{A}{\left(1+\left|b_{s}\right|\right)^{2}} d s\right)=+\infty
$$

car déjà

$$
\mathrm{E}_{0}\left(\int_{0}^{+\infty} \frac{A}{\left(1+\left|b_{s}\right|\right)^{2}} d s\right)=+\infty
$$


Si nous revenons à $\boldsymbol{R}^{+}$par le changement de fonction usuel on a l'équation $v^{\prime \prime}+\frac{A}{x^{\prime 2}} v$ où $x^{\prime}=1+x$ dont les solutions d'énergie $-k^{2}$ sont

$$
\sqrt{\frac{\pi}{2} k(x+1)} J_{v}(k(x+1))
$$

où $v=\frac{1}{2} \sqrt{1-4 A}$ et $J_{v}$ est la fonction de Bessel. Bornons-nous au cas $A<1 / 4$; les solutions à énergie nulle sont dans $\boldsymbol{R}^{3}$

$$
v_{ \pm}(x)=\frac{(1+|x|)^{(1 / 2) \pm v}}{|x|}
$$

Alors $M_{t}^{ \pm}=\exp \left(\int_{0}^{t} \frac{A}{\left(1+\left|b_{s}\right|\right)^{2}}\right) v_{ \pm}\left(b_{t}\right)$ est toujours une martingale locale $>0$ si $A \leqq 1 / 4$ et par suite

$$
\mathrm{E}_{x}\left(M_{\infty}^{ \pm}\right) \leqq v_{ \pm}(x)
$$

Par suite, trajectoire par trajectoire, on a

$$
\operatorname{Exp}\left(\int_{0}^{t} \frac{A}{\left(1+\left|b_{s}\right|\right)^{2}} d s\right) \sim C t^{(1 / 4)+(v / 2)}
$$

$C$ étant une constante dépendant de la trajectoire.

Examinons rapidement le cas des densités spectrales; pour ce potentiel particulier, elles se calculent directement en utilisant la formule (1.10) car les fonctions $y_{\lambda}^{(1)}$ sont connues; on a alors trois cas:

1) $A<1 / 4$

$$
\frac{d \rho\left(k^{2}\right)}{d\left(k^{2}\right)}=\frac{C(v)}{\left|H_{v}^{(1)}(k)\right|^{2}}
$$

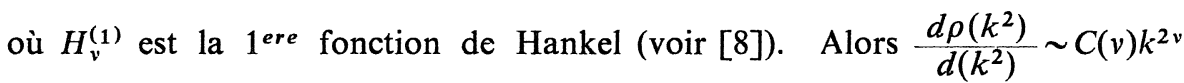
si $k \rightarrow 0$. On voit donc que on peut obtenir ici n'importe quel exposant $v$ pourvu que la constante de couplage soit bien choisie contrairement au cas des potentiels à courte portée. En particulier, utilisant (1.9) bis et le théorème abélien on déduit le comportement de $(\exp (L t) f \mid f)$ pour $t \rightarrow+\infty$ qui est en $t^{-2 v}$.

2) Pour $A=1 / 4 \quad \frac{d \rho\left(k^{2}\right)}{d\left(k^{2}\right)}=\frac{C(v)}{\left(\log k^{2}\right)^{2}}$.

3) Enfin pour $A>1 / 4, v$ devient imaginaire pur; écrivons $v=i v_{0}$ où $v_{0}$ est réel positif; alors $d \rho\left(k^{2}\right) / d\left(k^{2}\right)$ a un comportement oscillatoire près de 0 en

$$
\left|\left(k^{2}\right)^{\pi v_{0}}-\left(k^{2}\right)^{\left(-2 i\left(v_{0} \log \left(\frac{k}{2}\right)-\theta(v)\right)\right)}\right|^{-2}
$$


(ici $C(v), \theta(v)$ sont des constantes ne dépendant que de $v$ ).

c) On peut également étudier

$$
q(r)=\frac{A}{(1+r)^{\theta}}+-\frac{B}{(1+r)^{2}}
$$

pour $\theta>2$, les solutions à énergie nulle dans $\mathbb{R}^{3}$ sont

$$
\frac{(v+|x|)^{1 / 2}}{|x|} J_{ \pm \frac{2}{2-\theta}} \sqrt{\frac{1}{4}-B}\left(\frac{2}{2-\theta} \sqrt{A}(1+|x|)^{\frac{2-\theta}{2}}\right)=v_{ \pm}(|x|) .
$$

Si $0<B<1 / 4$ et $2 /(2-\theta) \sqrt{A}<z_{0}$ où $z_{0}$ est le premier zéro de la fonction de Bessel considérée, on a une solution positive à énergie nulle et un comportement asymptotique bien défini; dans ce cas si l'on fabrique à l'aide de (6.3), (6.4) la martingale $M_{t}^{ \pm}$on aura encore qu'elle a une limite $M_{\infty}^{ \pm}$avec

$$
\mathrm{E}_{x}\left(M_{\infty}^{ \pm}\right)<v^{ \pm}(x) .
$$

Ce qui donne une sorte de borne (peu explicite) sur la croissance si $t \rightarrow+\infty$ de l'exponentielle de Kac.

\section{Références}

[1] Berthier, A. M. et Gaveau, B., Critère de convergence des fonctionnelles de Kac et applications en mécanique quantique et en géométrie, J. functional analysis, 29 (1978), 416-424.

[2] Doob, J., Stochastic processes, John Wiley, New York, 1953.

[3] Gaveau, B., Fonctions propres et non existence absolue d'états liés pour certains systèmes quantiques, Comm. Math. Physics, 69 (1979), 131-146.

[4] Gaveau, B. et Mazet, E., Physique mathématique. - Divergence des fonctionnelles de Kac et diffusion quantique, Comptes Rendus Acad. Sci. Paris, Ser. A, 3 (1980), 559-562.

[5] Kac, M., On some connections between probability theory and differential and integral equations, Proceedings of $2^{\text {nd }}$ Berkeley Symposium on Probability and Statistics, 1950, 189-215.

[6] —-, Probabilistic methods in scattering theory, Rocky Mountain Math. J., 4 (1974), 511-537.

[7] Vauthier, J., Fonctions de Green de $-\Delta+V$ et zéros des fonctions propres.

[8] Watson, G. N., A treatise on the theory of Bessel functions, Cambridge Univ. Press, Cambridge; Macmillan, New York, 1944. 
